\title{
Expression of Neural and Neurotrophic Markers in Nucleus Pulposus Cells Isolated from Degenerated Intervertebral Disc
}

\author{
Stefania E. Navone, ${ }^{1}$ Giovanni Marfia, ${ }^{1,2}$ Laura Canzi, ${ }^{1}$ Emilio Ciusani, ${ }^{3}$ Alessandra Canazza, ${ }^{1}$ Sergio Visintini, ${ }^{4}$ \\ Rolando Campanella, ${ }^{5}$ Eugenio A. Parati ${ }^{1}$ \\ ${ }^{1}$ The Cellular Neurobiology Laboratory, Cerebrovascular Diseases Unit, IRCCS Foundation Neurological Institute "C. Besta", via Celoria, 11 \\ 20133 Milan, Italy, ${ }^{2}$ Cell Therapy and Clinical Pharmarcology, Department of Medicine, Surgery and Dentistry, University of Milan, Milan Italy, \\ ${ }^{3}$ Laboratory of Clinical Investigation, IRCCS Foundation Neurological Institute "C. Besta", Milan, Italy, ${ }^{4}$ Neurosurgery Department, IRCCS \\ Foundation Neurological Institute "C. Besta", Milan, Italy, ${ }^{5}$ Neurosurgery Department, S. Carlo Borromeo Hospital, Milan, Italy
}

Received 8 September 2011; accepted 2 February 2012

Published online 28 February 2012 in Wiley Online Library (wileyonlinelibrary.com). DOI 10.1002/jor.22098

ABSTRACT: Intervertebral disc (IVD) degeneration is a common disorder of the lower spine. Since it is caused by loss of cellularity, there is interest in the comprehension of the cellular phenotypes. This study aimed to verify if stem cells isolated from nucleus pulposus of intervertebral discs (NPs-IVD), which may express neurogenic properties, may be implicated in IVD disease. NPs-IVD isolated from 14 human pathological discs were cultured under mesenchymal and neural differentiation. An induction of the neural markers GFAP, $\mathrm{NF}, \mathrm{MAP} 2$, O4, and a decrement of the expression of the immature neural markers $\beta$-tubulin III, Nestin, NG2, occurred within the neural differentiation. The expression of TrkA and p75NGFR, the receptors of NGF, was not correlated with neural induction; in contrast, TrkB, the BDNF receptor, increased and was co-expressed with acid sensing ion channel 3 (ASIC3). In the same condition, neuroinflammatory markers were over-expressed. We confirm our hypothesis that stem cells within IVD degeneration acquire neurogenic phenotype, causing the induction of markers related to inflammatory condition. These cells could promote the enrolment of neurotrophines in adaptation to the acidic microenvironment in degenerative conditions. These data could improve our knowledge about IVD cellularity and eventually lead to the development of pharmacological therapies. (c) 2012 Orthopaedic Research Society. Published by Wiley Periodicals, Inc. J Orthop Res 30:1470-1477, 2012

Keywords: intervertebral disc; nucleus pulposus cells; mesenchymal stem cells; neural differentiation; neuroinflammation

Low back pain is an extremely common symptom that could affect nearly three-quarters of the population sometime in their life. Ninety percent of the population recovers within 3 months, but in some patients chronic low back pain leads to long term physical disability and a reduced quality of life. ${ }^{1}$ The mechanism of disc degeneration is thought to be caused by the inability of the nucleus pulposus (NP) to maintain, after stress conditions, a normal content of proteoglycans and collagen, which are responsible for the trapping of water via osmosis and negative charge. ${ }^{2}$ Studies based on the characterization of the cells resident in the niche of the intervertebral disc (IVD) have improved our knowledge of the role of these cells and of their plasticity. ${ }^{3}$

Risbud and colleagues ${ }^{4}$ found evidence of skeletal progenitor cells in the degenerated cervical human IVD, suggesting a role in orchestrating the repair after degeneration. Despite this knowledge, all the types of cells that populate the IVD and their role in degenerative and regenerative process have yet to be identified. In addition, it is also unknown if the immunophenotypic and molecular properties, the proliferation and the differentiation abilities of these cells in pathological conditions are defective. Moreover, NP cells are in hypoxic conditions and cellular respiration is largely anaerobic. The consequence of this anaerobic

Correspondence to: Stefania E. Navone (T: 0039-02-23942722; F: 0039-02-23942722; E-mail: stefania.navone@istituto-besta.it; neurofarmacologia1@istituto-besta.it)

(c) 2012 Orthopaedic Research Society. Published by Wiley Periodicals, Inc. microenvironment is the presence of low $\mathrm{pH} .{ }^{5}$ In neural tissue, the adaptation to an acid niche is regulated by the activation of acid sensing ion channel (ASIC) proteins, ${ }^{6}$ that are implicated in pain transduction associated with ischemic or inflamed tissue acidosis ${ }^{7}$ as in NP of IVD. ${ }^{8}$ ASIC3 is a voltage-independent, depolarizing cationic channel mainly permeable to $\mathrm{Na}+$ ions $^{9}$ that opens when $\mathrm{pH}$ drops in the extracellular environment. ${ }^{10}$ The channel has been associated with inflammatory pain: mice lacking the ASIC3 channel do not develop chronic muscle pain from repeated administration of an acidic saline ${ }^{11}$ Experiments on rats suggest that the activity of this channel protein may serve to induce the IVD cells to express markers related to hyperinnervation to adapt to the altered environment in pathological conditions. ${ }^{12}$ Among the molecules that are presumably involved in IVD hyperinnervation, of great importance are the members of the neurotrophins (NTs) family. ${ }^{13}$ NTs enhance the survival and differentiation of different populations of peripheral nerve neurons. Nerve growth factor (NGF) and brain-derived neurotrophic factor (BDNF) have been found to be increased in degenerated IVD. ${ }^{14}$ Moreover, degenerative conditions induce the expression of inflammatory molecules that could modify the perception of pain. Calcitonin gene related peptide (CGRP), an inflammatory neuropeptide associated with pain and inflammatory state, is expressed on IVD. ${ }^{15}$ The purpose of this study was to investigate the features, the differentiation ability, and the role of NPs-IVD in degeneration process by the expression of markers involved in degenerative damage. 


\section{METHODS}

Isolation of Nucleus Pulposus Cells From Human Degenerated IVD (NPs-IVD)

The study protocol was approved by our institutional ethics committee and patients' informed consents to the procedure were obtained. In our study, samples of IVD were collected from the lumbar column of 14 patients undergoing surgeries. The samples were taken from 7 male and 7 female patients with similar degenerative disc case histories. Lumbosacral MRI of each patient was taken to assess Thompson degeneration grade. Samples, ranging between 3 and $6 \mathrm{~g}$, of degenerated disc were removed, nucleus pulposus (NP) was separated from the annulus fibrosus (AF) using a stereotaxic microscope, collected and placed in a falcon with Stem Cells Medium (SCM), as previously described, ${ }^{16}$ supplemented with $10 \%$ of fetal bovine serum (Gibco, Grand Island, NY) and $1 \%$ penicillin and streptomycin solution (Sigma-Aldrich, Basel, Switzerland). The tissues were maintained at $4{ }^{\circ} \mathrm{C}$ overnight, then were enzymatically digested for $16 \mathrm{~h}$ by means of Dulbecco's medium (Gibco) supplemented with $0.25 \%$ Liberase Blendzyme2 $(2.5 \mathrm{mg} / \mathrm{ml}$, Roche Diagnostics, Indianapolis, IA) in D-PBS, Dulbecco's phosphate buffered saline (Euroclone, Milan, Italy). The digested tissue/cell suspension was passed through a $100 \mu \mathrm{m}$ cell strainer (Falcon, Becton Dickinson, Allschwil, Switzerland) to remove tissue debris and centrifuged at $400 \mathrm{~g}$ for $10 \mathrm{~min}$. Human disc cells were seeded in $75-\mathrm{cm}^{2}\left(2 \times 10^{5}\right.$ cells $\left./ \mathrm{cm}^{2}\right)$ and weekly were detached from the tissue culture plates using Tryple Select (Gibco). NPs-IVD were expanded for experimental uses $\left(2 \times 10^{4}\right.$ cells $\left./ \mathrm{cm}^{2}\right)$ until at passage $10(\mathrm{P} 10)$ in a humidified atmosphere containing $5 \% \mathrm{CO}_{2}$ at $37^{\circ} \mathrm{C}$. Cell viability was assessed by Trypan Blue dye-exclusion assay.

\section{Analysis of Apoptosis}

To detect apoptosis in NPs-IVD, a DeadEND fluorometric TUNEL analysis (Promega, Madison, WI) was performed according to manufacturer's recommendations.

\section{Flow Cytometric Immunophenotyping}

For each sample, $5 \times 10^{4}$ cells were characterized by means of FACS. Cells were incubated with appropriate phycoerytrine (PE) or fluorescein isothiocyanate (FITC) conjugated antibodies to test the expression of a pattern of hematopoietic and mesenchymal markers: CD14, CD24, CD44, CD45, CD73, CD166 (BD Pharmingen, San Jose, CA), CD133/2, CD34 (Miltenyi Biotec, Bisley, Surrey, UK), CD105, (AbDSerotec, Raleigh, NC), and CD90 (Millipore, Temecula, CA). After $30 \mathrm{~min}$ at $4^{\circ} \mathrm{C}$, cells were washed once with PBS, fixed with $4 \%$ paraformaldehyde (PFA), and were analyzed using a
FACS scan flow cytometer and Cell Quest software (BD Pharmingen). Isotype-matched mouse immunoglobulins were used as control.

\section{Differentation Assay}

Human Mesenchymal Stem Cell Functional Identification Kit (R\&D Systems, Minneapolis, MN) was used to induce adipogenic, chondrogenic, and osteogenic differentiation. According to the kit, the expression of adipogenic, osteogenic, and chondrogenic markers was tested, respectively, with goat anti-mouse FABP-4 antibody, with mouse anti-human osteocalcin antibody, and with goat anti-human aggrecan antibody as reported in the procedure. Negative controls were made to evaluate the specificity of the antibodies. Moreover, NPs-IVD were plated in 4-well chamber slides coated with collagen bovine type IV (BD Pharmingen) in the NEU medium, the culture medium (SCM) deprived by mitogens (EGF and bFGF) which facilitates the expansion of neural cells, and $10 \%$ of fetal bovine serum (Gibco) for 2 weeks and fixed in $4 \%$ PFA. The positive expression for neural markers was analyzed by means of immunostaining with lineage-specific antibodies: GFAP (rabbit, 1:500), NG2 (rabbit, 1:200), $\beta$-III tub (mouse, 1:100), neurofilament, NF-150 KDa (rabbit, 1:100) (Chemicon, Temecula, CA); Nestin (mouse, 1:100; R\&D systems), MAP2 (mouse 1:100), and oligodendrocyte marker O4 (mouse 1:20) (Boehringer-Mannheim, Indianapolis, IN). TrkA (rabbit 1:100), p75NGFR (rabbit 1:500) AbCam, Cambridge, MA), and TrkB (mouse 1:100) (Millipore) were utilized to detect the expression of neurotrophins markers. ASIC3 (goat 1:100, R\&D System), Sodium Channel-pan (rabbit 1:100, Novus Biologicals, Littleton, CO) and CGRP (mouse 1:100, AbCam) immonostainings were carried out to evaluate degeneration condition. After three washings nuclei were counterstained with DAPI $(1 \mu \mathrm{g} / \mathrm{ml})$ (Chemicon), the cells were incubated with appropriate secondary antibody and finally were mounted with Fluorsave ${ }^{\mathrm{TM}}$ Reagent (Calbiochem, Darmstadt, Germany) and viewed under a Zeiss Axiophot-2 microscope. Specificity of the secondary antibodies was confirmed by negatively staining cells with the secondary antibodies alone, with no staining of $>98 \%$ of the total cells.

\section{Gene Expression Analysis}

Three separate RNA extractions were performed on NPs-IVD and processed separately. Total RNAs from each cell line were isolated with TRIzol reagent (Invitrogen, Carlsbad, CA) according to the manufacturer's protocol. cDNA was prepared from $1 \mu \mathrm{g}$ RNA using SuperScript First- Strand Synthesis Kit (Invitrogen). PCR amplification primer pairs were bought from Proligo Sigma (St. Louis, MO; Table 1). The

Table 1. Primers Utilized in PCR Analysis

\begin{tabular}{|c|c|c|c|c|}
\hline & Primer & $\mathrm{bp}$ & $\mathrm{Ta}\left({ }^{\circ} \mathrm{C}\right)$ & Cycles \\
\hline NGF & $\begin{array}{l}\text { Forward: CTG GCC ACA CTG AGG TGC AT } \\
\text { Reverse: TCC TGC AGG GAC ATT GCT CTC }\end{array}$ & 120 & 53 & 40 \\
\hline BDNF & $\begin{array}{l}\text { Forward: AAA CAT CCG AGG ACA AGG TG } \\
\text { Reverse: AGA AGA GGA GGC TCC AAA GG }\end{array}$ & 248 & 55 & 35 \\
\hline ASIC3 & $\begin{array}{l}\text { Forward: TAT GAG ACC GTG GAG CAG } \\
\text { Reverse: TGT GTG ACA AGG TAG CAG }\end{array}$ & 331 & 58 & 30 \\
\hline GAPDH & $\begin{array}{l}\text { Forward: CGG AGT CAA CGG ATT TGG TCG TAT } \\
\text { Reverse: AGC CTT CTC CAT GGT GGT GAA GAC }\end{array}$ & 309 & 58 & 35 \\
\hline
\end{tabular}

bp, base pair; Ta, temperature of annealing in degree Celsius $\left({ }^{\circ} \mathrm{C}\right)$. 
PCR products were visualized on ethidium bromide-stained $2 \%$ agarose gels and evaluated by transilluminator.

\section{Statistical Analysis}

The right number of replicates for each experiment in vitro was determined on the basis of the inter- and intra-assay variation (from $1 \%$ and $5 \%$ ) of the single assay and the standard deviation of the results was shown. In Real Time a $p$ value of $<0.05$ was considered significant. We repeated each experiment at least three times.

\section{RESULTS}

\section{Isolation of NPs-IVD}

Cells isolated from NP of 14 human degenerated IVD samples after 1 day of culture in SCM supplemented with 10\% FBS showed initially a neurosphere-like morphology. After 14 days in vitro, some cells displayed floating macroaggregates while other cells demonstrated polygonal morphology and during passages, monolayer cultured cells remained adherent; however assuming a neural/astrocyte like cells morphology (Fig. 1A). The apoptotic analysis with TUNEL test revealed that a small number of cells $(7.23 \pm 2.5 \%)$ showed the degradation of DNA in membrane blebbing (Fig. 1B). The percentage of apoptotic cells was calculated by FACS analysis with Annexin V. Moreover, a double staining procedure with propidium iodide was performed to distinguish apoptotic from necrotic cells. In fact, apoptotic cells were Annexin $\mathrm{V}+$ /propidium iodide- $(6.51 \pm 3.80 \%)$, while necrotic cells were Annexin $\mathrm{V}+$ /propidium iodide+ $(0.01 \pm 0.01 \%)$ (Fig. 1C).

A
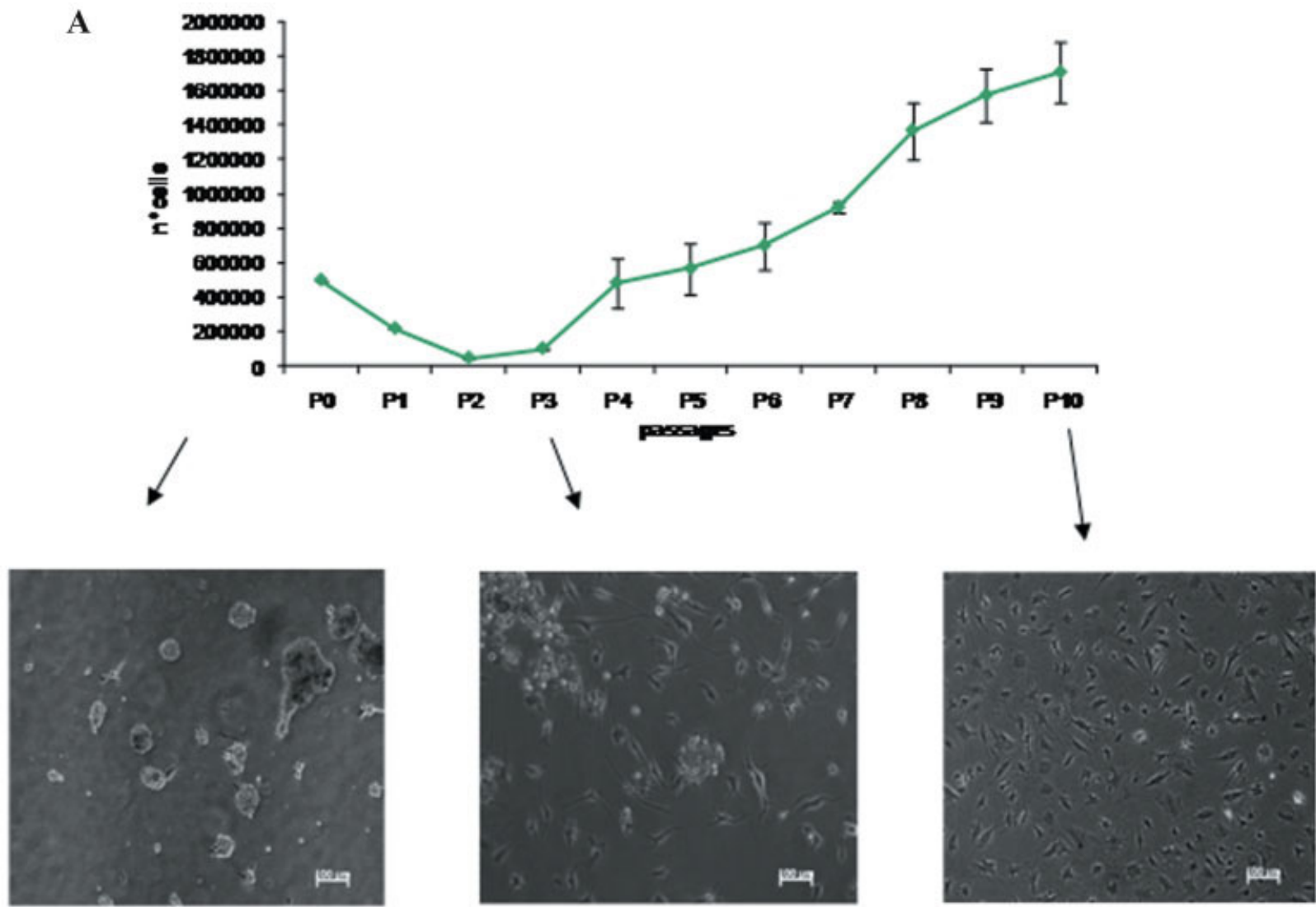

B

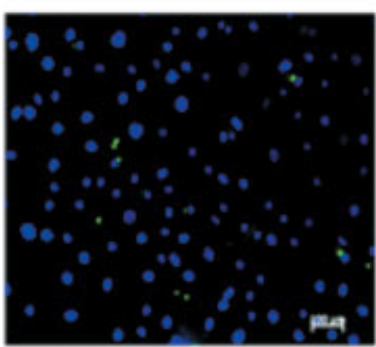

C

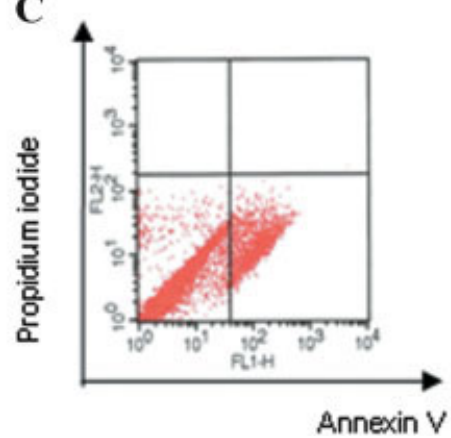

Figure 1. Isolation of NPs-IVD: (A) Growth curve and photographs of NPs-IVD morphology in different time of culture. (B) TUNEL test and (C) FACS analysis revealed the low percentage of apoptotic and necrotic cells. 
NPs-IVD Express Mesenchymal Markers and Differentiation Properties

Immunophenotypic FACS analysis showed that the NPs-IVD population, kept in culture in SCM, expressed high percentage of positivity for CD24, CD44, CD73, CD90 CD105, and CD166, a lower positivity fraction for CD34, they were negative for CD14 and for CD45 and the expression of CD133 was unconsiderable (0.59\%) (Fig. 2A). Adherent NPs-IVD were cultured for 14 days under conditions that promoted the differentiation towards chondrogenic, adipogenic, and osteogenic lineages. Immunofluorescence analyses of NPs-IVD were performed to analyze the expression of lineage specific markers. NPs-IVD cultured in chondrogenic medium, expressed positivity for aggrecan (93.81 $\pm 5.52 \%)$, a marker used to evaluate deposition of proteoglycans.
When treated with adipogenic medium, fatty acid binding protein 4 (FABP-4) expressions was $84.30 \pm$ $6.85 \%$. Moreover, NPs-IVD acquire osteocalcin marker $(92.65 \pm 4.48 \%)$ (Fig. 2B).

\section{NPs-IVD Differentiate Into a Neurogenic Phenotype}

To study neurogenic differentiation, NPs-IVD were cultured on collagen coated chamber slides in NEU medium supplemented with $10 \%$ of fetal bovine serum for 14 days. The positive expression of NPs-IVD for neural markers was analyzed by means of immunostaining with neural lineage-specific antibodies and a comparison with NPs-IVD cultured in basal medium (SCM) was done (Fig. 3A). NP-IVDs, already highly positive for $\beta$ tub III $(75.45 \pm 11.80 \%)$ and nestin $(67.62 \pm$ $14.50 \%$ ) in basal condition, under differentiation stimuli,

A

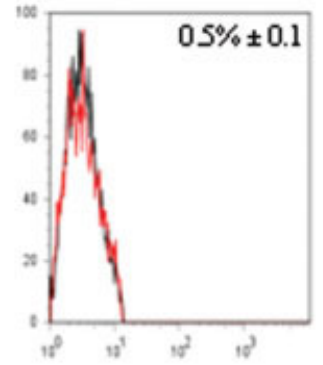

CD14PE

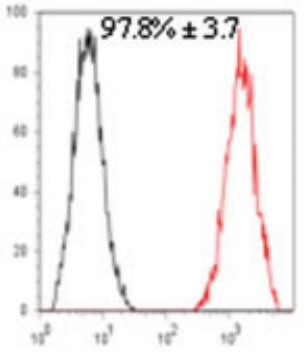

CD73-PE

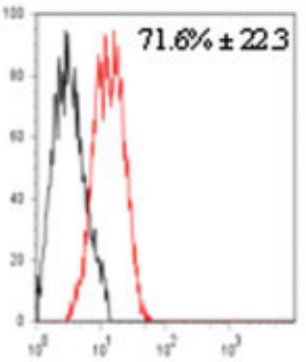

CD24PE

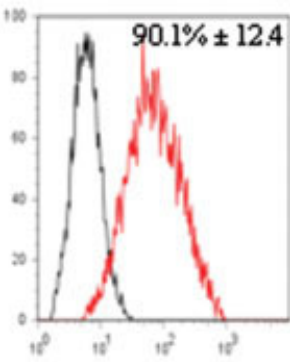

CD90-PE

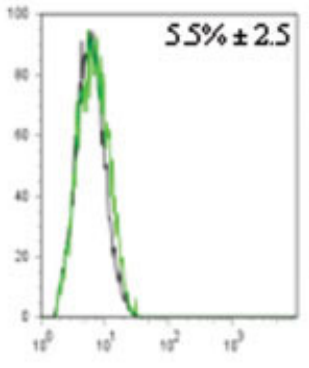

CD34-FITC

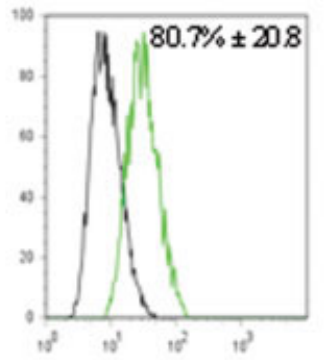

CD105-FITC

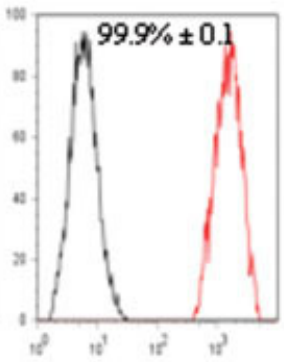

CD44PE

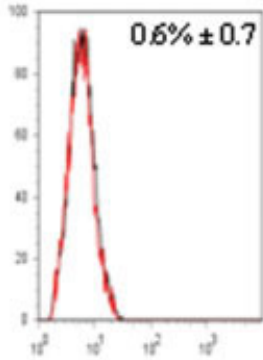

CD133-PE

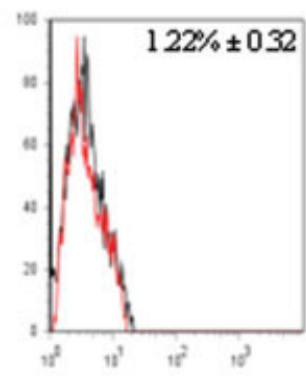

CD45-PE

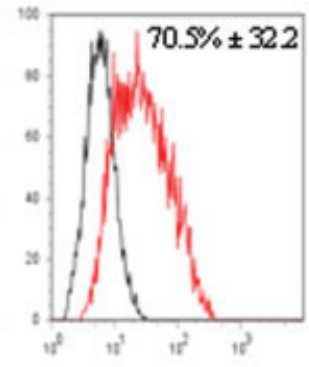

CD166-PE

B
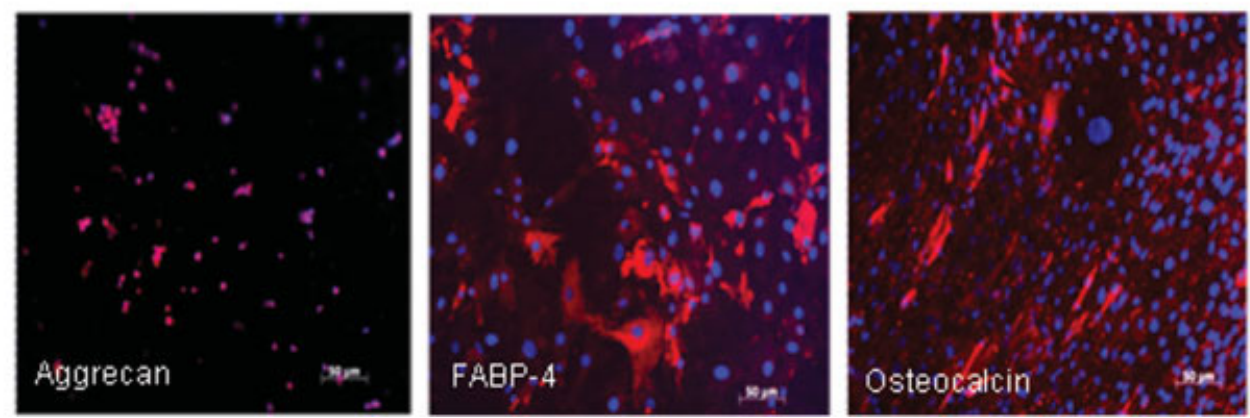

Figure 2. Mesenchymal Profile of Human NPs-IVD: (A) Immunophenotypic profile, by citofluorimetric analysis, of nucleus pulposus from 14 human degenerated intervertebral discs. (B) NPs-IVD show mesenchymal properties under chondrogenic, adipogenic, and osteogenic differentiation. 
A

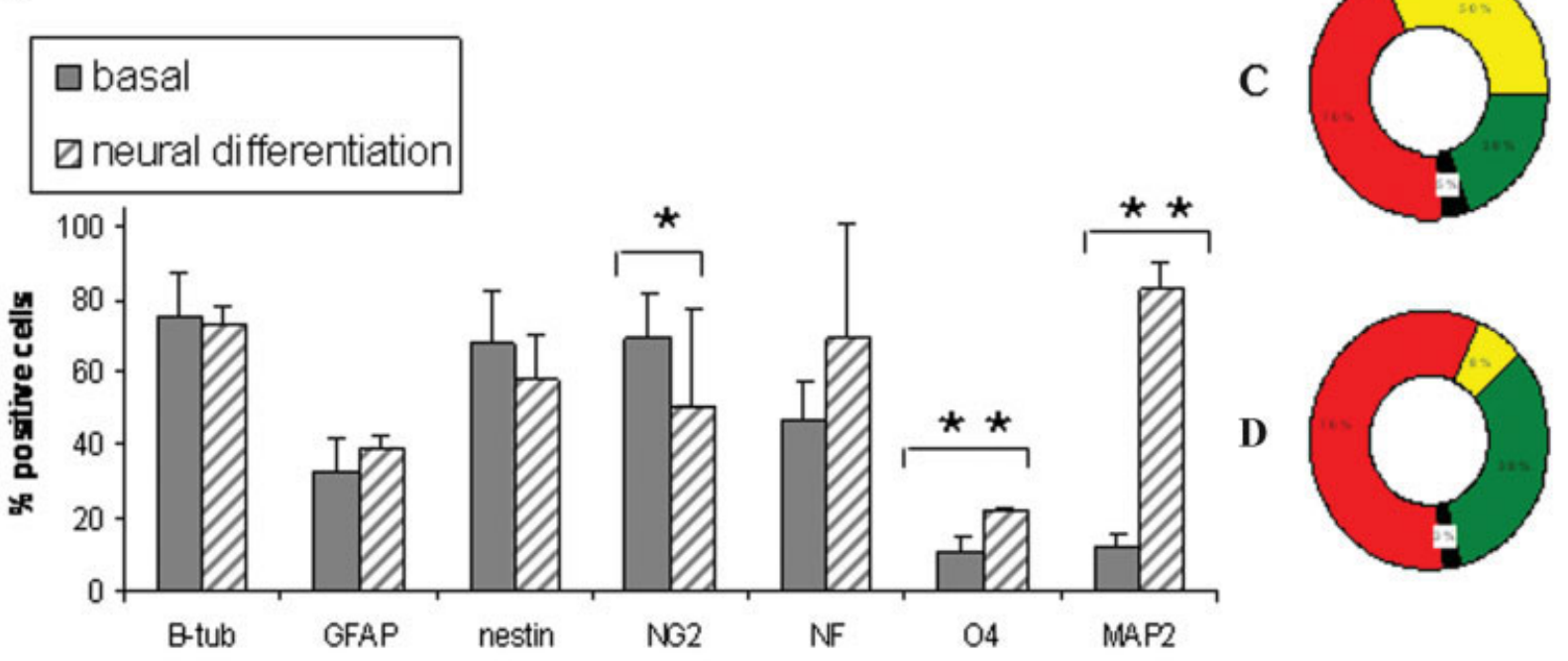

B
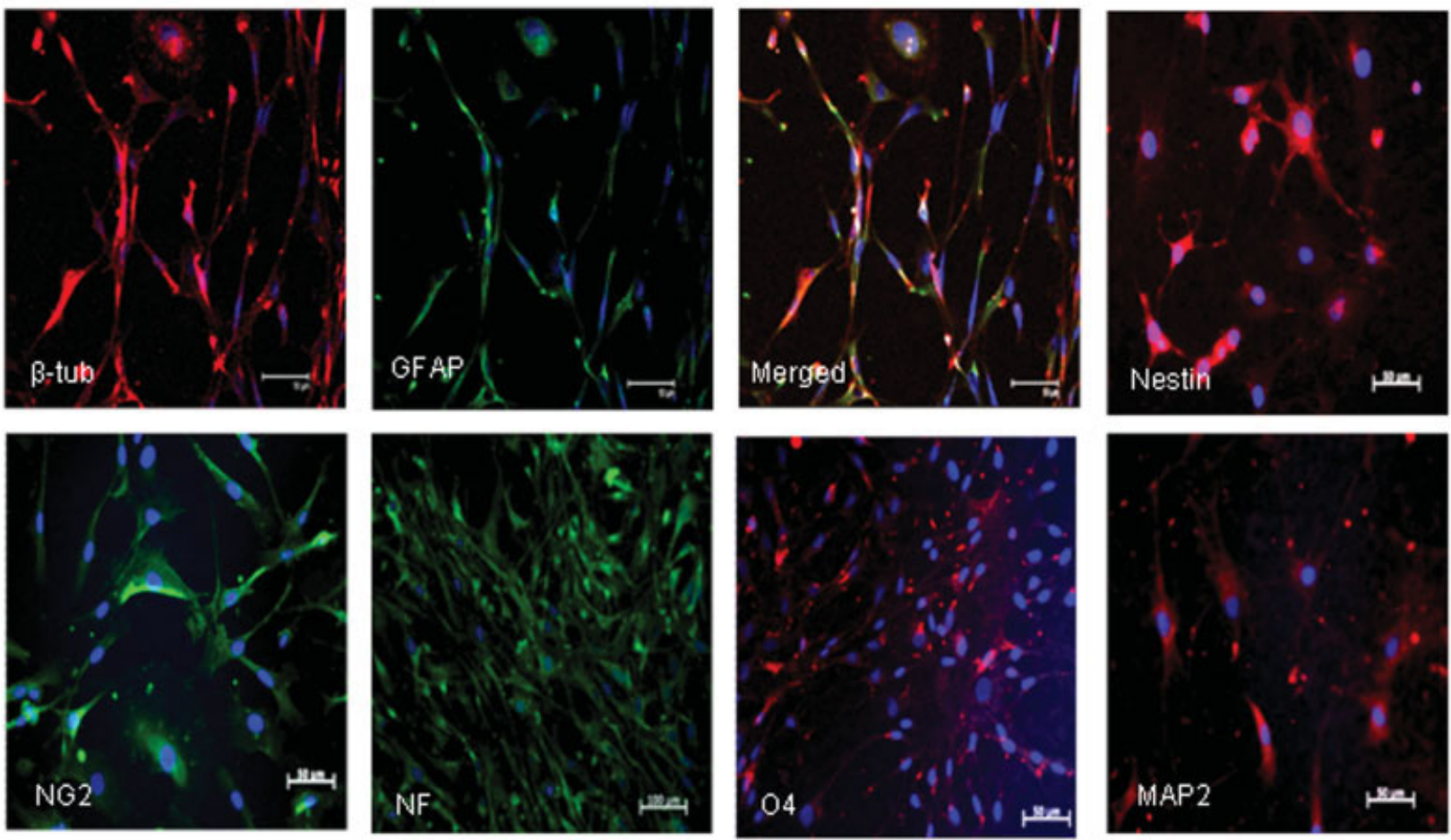

Figure 3. Neurogenic phenotype of NPs-IVD: (A) Histograms show the \% of positive cells with standard deviation expressing immature and mature neural markers in basal condition (SCM) and under neural differentiation (NEU medium) examined by immunofluorescence analysis. ${ }^{*} p<0.05 ;{ }^{* *} p<0.01$. (B) Neural markers immuno-reactivity. (C,D) The ring charts show the relative distribution of the co-localization $\beta$-tub III and GFAP in basal and neural condition. Black part corresponds to the cells that do not express the mentioned markers; yellow part corresponds to the cells that co-localized the two markers. [Color figure can be seen in the online version of this article, available at http://wileyonlinelibrary.com/journal/jor]

become highly positive also for GFAP $(39.29 \pm 3.29 \%)$, NF $\quad(69.14 \pm 31.43 \%), \quad$ NG2 $\quad(50.91 \pm 26.90 \%), \quad$ O4 $(22.36 \% \pm 0.65 \%)$, and MAP2 (83.01 $\pm 6.91 \%)$ (Fig. 3B).

In addition, the cell population cultured in SCM medium, co-expressed with high positivity $\beta$-tub III and GFAP ( $\beta$-tub III/GFAP $51.93 \pm 3.37 \%$ ) (Fig. 3C); while, upon differentiation conditions, a modest coexpression $(14.3 \pm 3.8 \%)$ was found within NPs-IVD (Fig. 3D). The expression of TrkA $(70.63 \pm 13.19 \%)$, p75NGFR $(74.01 \pm 19.52 \%)$, and TrkB $(98.5 \pm 1.20 \%)$ were also evaluated. The analysis of ASIC3, TrkA, and TrkB showed a great number of cells that co-localized. Almost 100\% of cell population positive for ASIC3, coexpressed with TrkA and TrkB (Fig. 4A,B). In neural differentiation conditions, the positivity for inflammatory markers Sodium Channel-pan $(87.24 \pm 15.41 \%)$ and CGRP $(73.95 \pm 12.76 \%)$ (Fig. 4C,D) was higher than in the basal condition. Moreover there was a colocalization between p75NGFR and CGRP (Fig. 4E). The expression level for TrkB is significantly elevated 
A

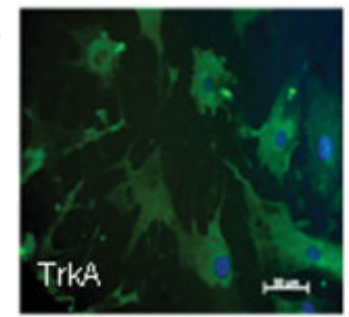

B

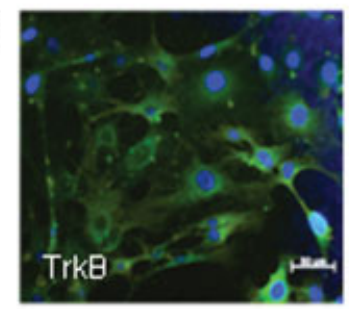

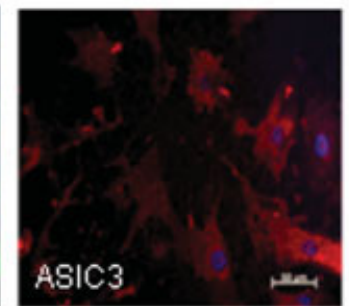

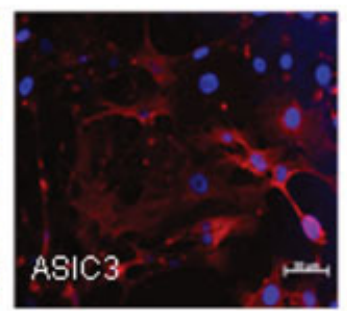

E

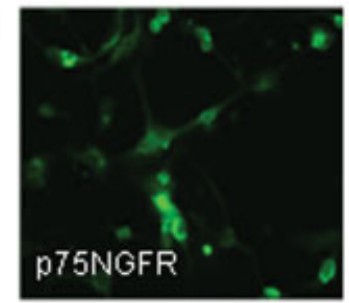

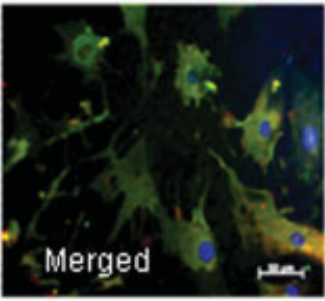
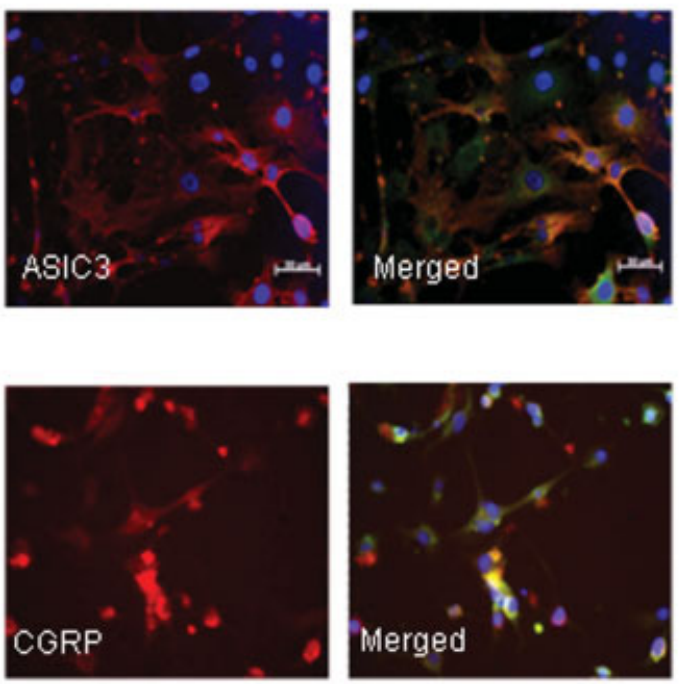
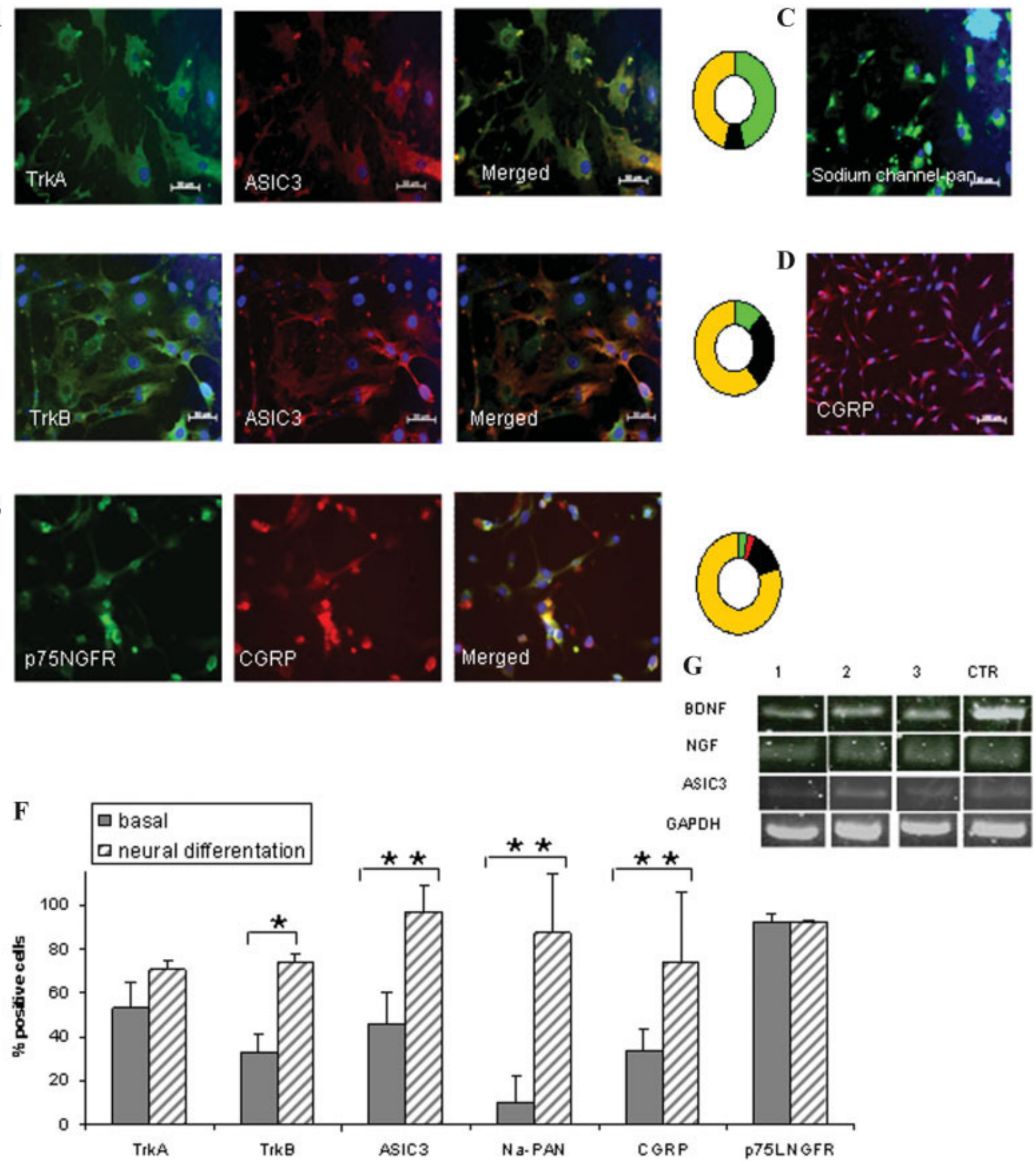

Figure 4. Expression of neurotrophic and neuroinflammatory markers: (A,B,E) NPs-IVD express markers related to the acidic microenvironment and an inflammatory state: TrkA and TrkB with colocalization with ASIC3, and p75NGFR with colocalization with CGRP. The ring charts show the single expression or the yellow double-labeling expression of the markers. Black part corresponds to the cells that do not express the mentioned markers. (F) Histograms show the \% of positive cells with standard deviation of neurotrophic and inflammatory markers related to basal and neural differentiation culture conditions ${ }^{*} p<0.05 ;{ }^{* *} p<0.01$. (C) Sodium Channel-pan and (D) CGRP expressions. (G) PCR analysis was performed in three samples $(n=1,2,3)$ of NP-IVDs of BDNF, NGF, and ASIC3 related to adult brain, the control sample. GAPDH was used as a housekeeping gene. [Color figure can be seen in the online version of this article, available at http://wileyonlinelibrary.com/journal/jor]

after neural differentiation, while no differences in the expression for TrkA and p75NGFR were found (Fig. 4F). Moreover, PCR analysis of neural differentiated NPs-IVD indicated that the cells expressed NGF, BDNF and ASIC3 mRNA. Similarly, NGF, BDNF, and ASIC3 mRNAs expression was found in the brain, the control sample (Fig. 4G).

\section{DISCUSSION}

In the present study we describe the characterization of the phenotype of NPs-IVD. Two major issues are addressed: 1) the identification of cells isolated from NP of human IVD with mesenchymal profile that can differentiate into neural phenotype; 2) degenerated NPs-IVD that, by the expression of ASIC3 and CGRP, 
could be responsible for hyperinnervation due to the acidic microenvironment contributing to the degeneration process. NPs-IVD express the majority of cell surface antigens expressed in mesenchymal cells, CD44, CD73, CD90, CD105, and CD 166 and show plastic adherent ability. ${ }^{17}$ They express CD24, a glycosylphosphatidylinositol anchor protein, a cell surface marker for NP, ${ }^{18}$ while they are less positive for CD34 and negative for CD14 and CD45, which mark primitive hematopoietic progenitors and endothelial cells. The expression of CD133, evaluated by FACS analysis, was inconsiderable $(0.59 \%)$, showing a phenotypic profile of mesenchymal stem cells. ${ }^{19}$ Moreover, these cells demonstrate a high level of multipotenciality as they could induce the expression of adipogenic, osteogenic, chondrogenic, and neural markers, in the presence of appropriate stimuli. Our study is comparable to the recent work of Feng and colleagues, who showed the expression of neurogenic markers in AF cells. They reported that cells from nondegenerative IVD of adolescent patients with idiopathic scoliosis, if cultured under media containing different stimuli, are able to express neural markers such as $\beta$-tub III, Nestin, and MAP2. ${ }^{20}$ It is important to highlight that our data refer to NP, the inner part of the IVD and that show an over-expression of neural and inflammatory markers in neural induction. Similarly to our data, the recent work published by del Cañizo et al., showed the presence of mesenchymal stem cells from NP of degenerated IVD. They identified a population of mesenchymal stromal cells able to differentiate into chondrogenic and osteogenic lineages. In contrast to our results they did not report the differentiation of their cells into an adipogenic lineage. ${ }^{3}$ Notably, the expression of Nestin has previously been demonstrated to have an important role in allowing MSC derived from adult bone marrow to commit to a neural and astrocytic fate. ${ }^{21}$ Here we show that Nestin is expressed in NPs-IVD, which could explain why these cells are able to express markers of neuronal, astrocytic, and oligodendrocytic lineages in differentiation condition. In addition, the cells cultured in basal condition, show co-expression of $\beta$-tub III and GFAP, suggesting that they could be in the progenitor stage (or glioblast) of neurogenic differentiation. ${ }^{22}$ The high expression of neurotrophins, such as $\mathrm{NGF}$ and $\mathrm{BDNF}$, resulting from immunohistochemical and molecular analyses, seems to show their important role in the degenerative condition when there is hyperinnervation phenomenon. Kirschenbaum and colleagues ${ }^{23}$ explained that in degenerated IVD there is an overexpression of BDNF that could promote the neural differentiation and survival of newly generated subventricular zone daughter cells. Yamauchi et al. ${ }^{13}$ described that NGF, from the $\mathrm{NP}$, promotes the growth of sensory nerve fibres innervating the degenerated IVD. Sugiura ${ }^{24}$ investigated the presence of TrkA and p75NGFR in a rat model, illustrating high expression of these two receptors in the superficial part of the AF. Moreover, Gruber and colleagues reported the presence of BDNF and its receptor mRNA in the human outer annulus, inner annulus, and nucleus pulposus of degenerated IVDs. ${ }^{25}$ Recently, the expression of mRNA of NGF was reported in the article of Ponnappan and colleagues. The authors showed the expression of NGF mRNA in a model of organ culture of degenerated IVD. ${ }^{26}$ This point is interesting because, as suggested, the expression of NT receptors is evident in basal and in neural differentiation conditions and it can be possible that the ligands expression have the same behaviour of their receptor after neural induction. Furthermore, additional molecular and pharmacological studies are needed to support this hypothesis. Purmessur and colleagues, ${ }^{27}$ investigated the expression of TrkA and TrkB in NP of healthy and degenerated IVD, associating their expression with the severity of disc degeneration in cells derived from the human IVD. As indicated in our results, they showed the immunopositivity for TrkA and TrkB in degenerated IVD. Moreover, our data illustrated the positive expression of TrkB that increased under neural differentiation. The significance of this increment could be explained by suggesting an autocrine effect on the neural disc cells themselves in degeneration conditions. Moreover, the co-localization of TrkA and TrkB sites banding proteins with ASIC3 suggests a possible correlation between the acidic environments with the hyperinnervation condition. Almost $100 \%$ of ASIC3-positive NPsIVD, express TrkA and TrkB receptors. The presence of the cells which co-express ASIC3 and TrkA or TrkB, could be explained by the fact that some cells are more responsive than others to the stress conditions of the microenvironment. Furthermore, our results evidence the over-expression of CGRP in neural differentiation. Recently, was reported that DRG neurons innervating punctured rats IVD were positive for CGRP and the administration of a toxin able to destroy p75NGFR, can induce the suppression of CGRP. Thus, p75NGFR may be an important receptor to mediate discogenic pain via CGRP expression. ${ }^{28}$ The origin of NPs-IVD within human degenerated IVD with neurogenic differentiation potential is not easy to explain. These cells may be derived from the vertebral column. The IVD, in fact, is derived from the notochorda and some notochordal cells remain in the adult IVD. ${ }^{29}$ It is possible that cells, quiescent for all their life, become active and subsequently express neurogenic properties in response to degeneration conditions. Probably, due to the acidic microenvironment, they are able to express neurotrophic factors in order to adapt to their unique, hydrodynamically degenerated condition.

A study of this nature is critical for better comprehension of the disease process. Isolation of stem cells from pathological IVD could represent a good tool to understand more clearly the pathological mechanisms that underlie IVD degeneration, to develop an in vitro 
model of IVD for pharmacological studies and eventually, to allow cell transplantation as a regeneration therapy.

\section{ACKNOWLEDGMENTS}

We are grateful to Dr. Andrea Smith for her kind, helpful co-operation for the English review of the manuscript. We also thank Dr. Giulio Alessandri for the critical reading of this scientific article. This work was supported by LR8 IRCCS Foundation Neurological Institute "C. Besta", Milan, Italy.

\section{REFERENCES}

1. Macfarlane GJ, Thomas E, Croft PR, et al. 1999. Predictors of early improvement in low back pain amongst consulters to general practice: the influence of pre-morbid and episoderelated factors. Pain 80:113-119.

2. Pearce RH, Grimmer BJ, Adams ME. 1987. Degeneration and the chemical composition of the human lumbar intervertebral disc. J Orthop Res 5:198-205.

3. Blanco JF, Graciani IF, Sanchez-Guijo FM, et al. 2010. Isolation and characterization of mesenchymal stromal cells from human degenerated nucleus pulposus: comparison with bone marrow mesenchymal stromal cells from the same subjects. Spine (PhilaPa 1976) 26:2259-2265.

4. Risbud MV, Guttapalli A, Tsai TT, et al. 2007. Evidence for skeletal progenitor cells in the degenerate human intervertebral disc. Spine (PhilaPa 1976) 23:2537-2544.

5. Risbud MV, Schipani E, Shapiro IM. 2010. Hypoxic regulation of nucleus pulposus cell survival: from niche to notch. Am J Pathol 176:1577-1583.

6. Deval E, Gasull X, Noël J, et al. 2010. Acid-sensing ion channels (ASICs): pharmacology and implication in pain. Pharmacol Ther 128:549-558.

7. Mamet J, Baron A, Lazdunski M, et al. 2002. Proinflammatory mediators, stimulators of sensory neuron excitability via the expression of acid-sensing ion channels. J Neurosci 22:10662-10670.

8. Uchiyama Y, Cheng CC, Danielson KG, et al. 2007. Expression of acid-sensing ion channel 3 (ASIC3) in nucleus pulposus cells of the intervertebral disc is regulated by p75NTR and ERK signalling. J Bone Miner Res 22:1996-2006.

9. Lingueglia E, Deval E, Lazdunski M. 2006. FMRFamidegated sodium channel and ASIC channels: a new class of ionotropic receptors for FMRFamide and related peptides. Peptides 27:1138-1152. Review.

10. Krishtal O. 2003. The ASICs: signaling molecules? Modulators? Trends Neurosci 26:477-483.

11. Sluka KA, Price MP, Breese NM, et al. 2003. Chronic hyperalgesia induced by repeated acid injections in muscle is abolished by the loss of ASIC3, but not ASIC1. Pain 106: 229-239.

12. Xiong ZG, Pignataro G, Li M, et al. 2008. Acid-sensing ion channels (ASICs) as pharmacological targets for neurodegenerative diseases. Curr Opin Pharmacol 8:25-32.

13. Yamauchi K, Inoue G, Koshi T, et al. 2009. Nerve growth factor of cultured medium extracted from human degenerative nucleus pulposus promotes sensory nerve growth and induces substance p in vitro. Spine (PhilaPa 1976) 34:22632269.

14. Huang EJ, Reichardt LF. 2003. Trk receptors: roles in neuronal signal transduction. Annu Rev Biochem 72:609-642. Review.

15. Benemei S, Nicoletti P, Capone JG, et al. 2009. CGRP receptors in the control of pain and inflammation. Curr Opin Pharmacol 9:9-14.

16. Alessandri G, Pagano S, Bez A, et al. 2004. Isolation and culture of human muscle derived stem cells able to differentiate into myogenic and neurogenic cell lineages. Lancet 364: 1872-1883.

17. Dominici M, Le Blanc K, Mueller I, et al. 2006. Minimal criteria for defining multipotent mesenchymal stromal cells. The International Society for Cellular Therapy position statement. Cytotherapy 8:315-317.

18. Fujita N, Miyamoto T, Imai J, et al. 2005. CD24 is expressed specifically in the nucleus pulposus of intervertebral discs. Biochem Biophys Res Commun 338:1890-1896.

19. Phuc PV, Ngoc VB, Lam DH, et al. 2011. Isolation of three important types of stem cells from the same samples of banked umbilical cord blood. Cell Tissue Bank [Epub ahead of print].

20. Feng G, Yang X, Shang H, et al. 2010. Multipotential differentiation of human anulus fibrosus cells: an in vitro study. J Bone Joint Surg Am 92:675-685.

21. Wislet-Gendebien S, Hans G, Leprince P, et al. 2005. Plasticity of cultured mesenchymal stem cells: switch from nestinpositive to excitable neuronlike phenotype. Stem Cells 233: 392-402.

22. Belachew S, Chittajallu R, Aguirre AA, et al. 2003. Postnatal NG2 proteoglycan-expressing progenitor cells are intrinsically multipotent and generate functional neurons. J Cell Biol 161:169-186.

23. Kirschenbaum B, Goldman SA. 1995. Brain-derived neurotrophic factor promotes the survival of neurons arising from the adult rat forebrain subependymal zone. Proc Natl Acad Sci 92:210-215.

24. Sugiura A, Ohtori S, Yamashita M, et al. 2008. Existence of nerve growth factor receptors, tyrosine kinase a and p75 neurotrophin receptors in intervertebral discs and on dorsal root ganglion neurons innervating intervertebral discs in rats. Spine (PhilaPa 1976) 1:2047-2051.

25. Gruber HE, Ingram JA, Hoelscher G, et al. 2008. Brainderived neurotrophic factor and its receptor in the human and the sand rat intervertebral disc. Arthritis Res Ther 10:R82.

26. Ponnappan RK, Markova DZ, Antonio PJ, et al. 2011. An organ culture system to model early degenerative changes of the intervertebral disc. Arthritis Res Ther 13:R171.

27. Purmessur D, Freemont AJ, Hoyland JA. 2008. Expression and regulation of neurotrophins in the nondegenerate and degenerate human intervertebral disc. Arthritis Res Ther 10:R99.

28. Sugiura A, Ohtori S, Yamashita M, et al. 2010. Effect of applying p75NTR saporin to a punctured intervertebral disc on calcitonin gene-related peptide expression in rat dorsal root ganglion neurons. J Orthop Sci 15:407-413.

29. Risbud MV, Shapiro IM. 2011. Notochordal cells in the adult intervertebral disc: new perspective on an old question. Crit Rev Eukaryot Gene Expr 21:29-41. 